\title{
An Efficient Approach for Fetal ECG Extraction
}

\author{
Abdullah Mohammed Kaleem \\ Department of Electronics \& Telecomm. Engg. \\ MPGI School of Engineering, Nanded \\ Maharashtra, INDIA
}

\author{
Rajendra D. Kokate \\ Department of Instrumentation Engineering \\ Government College of Engineering, Jalgaon, \\ Maharashtra, INDIA
}

\begin{abstract}
Fetal ECG contains precise knowledge that may aid doctor in creating well-suited choices throughout pregnancy and labor. Authentic FECG signal is still extraordinarily complicated and very contaminated by outer disturbances. Hence extraction of clean fetal ECG is extraordinarily crucial for fetal surveillance. This is often accomplished by putting electrodes on mother's abdomen. Anyway it is tainted with varied sources of noise. This paper compares LMS adaptive filter for FECG extraction with neural network based adaptive filter. Real fetal ECG database was used. Experimental results validated superiority of later scheme in terms of SNR and MSE.
\end{abstract}

\section{General Terms}

Extraction, Algorithm, Comparison et.al.

\section{Keywords}

Fetal ECG, abdominal ECG, adaptive filter, neural network

\section{INTRODUCTION}

The electrocardiogram (ECG) shows sensible action of heart. The Fetal electrocardiogram (ECG) waveform examination is finished by the way of estimating electrical action from the fetal heart. The Fetal ECG (FECG) signal affords helpful information on physiological state of fetal heart. The FECG signals are often procured by 2 procedures, specifically fetal scalp electrode and maternal abdomen skin electrode [1]. Different methods have been forth put for fetal ECG extrication for instance matching pursuits[2], Independent component analysis(ICA) [3-5],blind source extraction(BSE)[6], adaptive filtering [7], support vector machine [8], singular value decomposition(SVD) [9], wavelet analysis [10], extended Kalman filtering [11], auto-correlation and cross-correlation techniques[12]. Adaptive filtering combined with neural network is a powerful scheme for FECG extraction. This paper demonstrates the proficiency of mentioned scheme over LMS based adaptive filter for FECG extraction by assessing Signal to Noise Ratio SNR and mean square error (MSE).

\section{ADAPTIVE NOISE CANCELLATION}

In fetal ECG extraction the Adaptive filter was utilized generally. Figure 1 shows the design of adaptive filter. Based on the streamlined preparing calculation the adaptive filter can modified. The streamlined preparing calculation was induced from the back proliferated blunder flag. In this method abdominal signal can be distinguished with the reference flag. And the qualification is named as mean Square error, which is to be limited. Adaptive filter is balanced by the mean square (LMS) calculation.

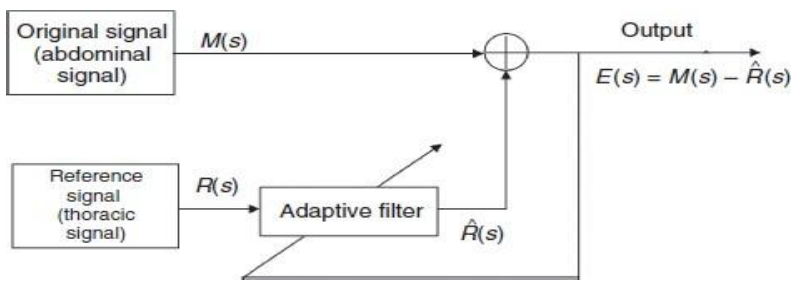

Fig 1: Adaptive filter

LMS update is given by

$$
w(n+1)=w(n)+2 \mu \mathrm{M}(\mathrm{S}) \mathrm{E}(\mathrm{S})
$$

Where $w(n)$ a vector comprising the coefficients of the FIR filters and $\mu$ is chosen step size.

For a FIR filter with $\mathrm{N}$ coefficients the output of the adaptive filter can be expressed as

$$
y(n)=W^{T}(n) x(n)
$$

Where $W=[w(0), w(1), \ldots w(m-1)]^{T}$

Fetal ECG can be obtained after adaptation as

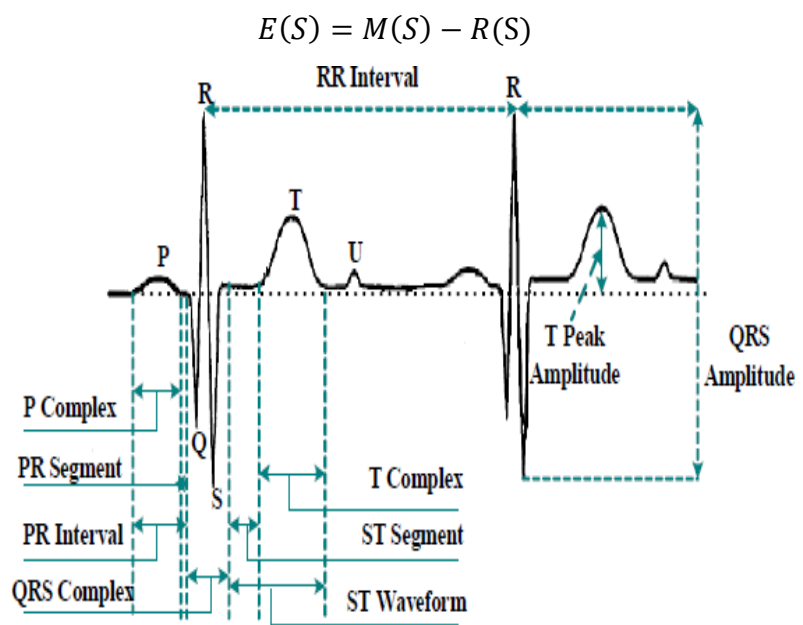

Fig 2: Typical ECG Waveform

3. NEURAL NETWORK BASED FILTER In this scheme the adaptive filter weights are ideally picked through two stage artificial neural network (ANN). Additionally, two stages ANN redesign the weight in perspective of the learning rate and force (weight of past cycle). By the help of two stages ANN the adaptive filter isolates the fetal flag through consequently gaining the appropriate parameters. The developed method using double stage ANN is appeared in figure 3. 
$M(S)=$ Abdominal signal;

$R(S)=$ Chest signal (Reference signal);

$d(s)=$ Delay with chest signal;

$d(s)=$ Delay with trained chest signal;

$E(S)=$ Error Signal (Extracted Fetal signal);

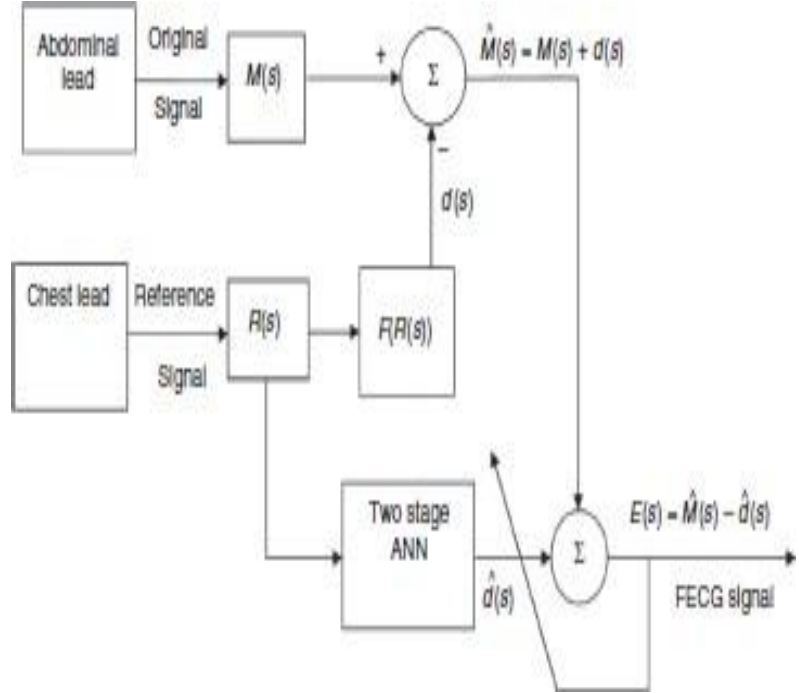

Fig 3: Adaptive filter with neural network

Abdominal signal and reference thoracic signals are selected as an input signals. The reference thoracic signal was denoted as $\mathrm{r}(\mathrm{j})$ and abdominal signal was denoted as $a$ (j) commonly $A$ (j) consist of noise source, which was denoted as $\mathrm{n} 0$ (j).

Likewise, the authentic input signal can be depicted as,

$$
M(S)=A(j)+n 0(j)
$$

Also, the reference input signal can be given as,

$$
R(S)=n 1(j)
$$

$n_{0}(\mathrm{j})$ and $n_{1}(\mathrm{j})$ are the noise sources, related to one another in some way that they ought to be equivalent. The $n_{0}(\mathrm{j})$ is an unidentified component. . To obtain the fetal ECG signal by removing the noise is a complicated progression. Input signal was related with the familiar noise source, $n_{1}(j)$ and the inequality is achieved by utilizing adaptive noise cancellation method. The dissimilarity yield (error signal) is the fetal ECG signal $f(j)$. In the two stage Artificial Neural Network strategy, the advancement is done as two stages. The ANN composed of three layers for instance, input layer, hidden layer and output layer. The reference maternal flag was given as contribution to the stage-I, where the reference maternal flag was created first with deferral. The execution of planning is evaluated by technique for the MSE. Stage - I neural framework yield was utilized to fabricate the stage-II neural system. For setting up, the output of Stage-I was given at the contribution of stage-II and the commitment of Stage-I is managed as the yield for Stage-II. By then the bipolar neural network (BPN) computation is implemented by utilizing this given information yield relationship. Finally, the Stage-II neural framework is organized up to the yield is refined without fault

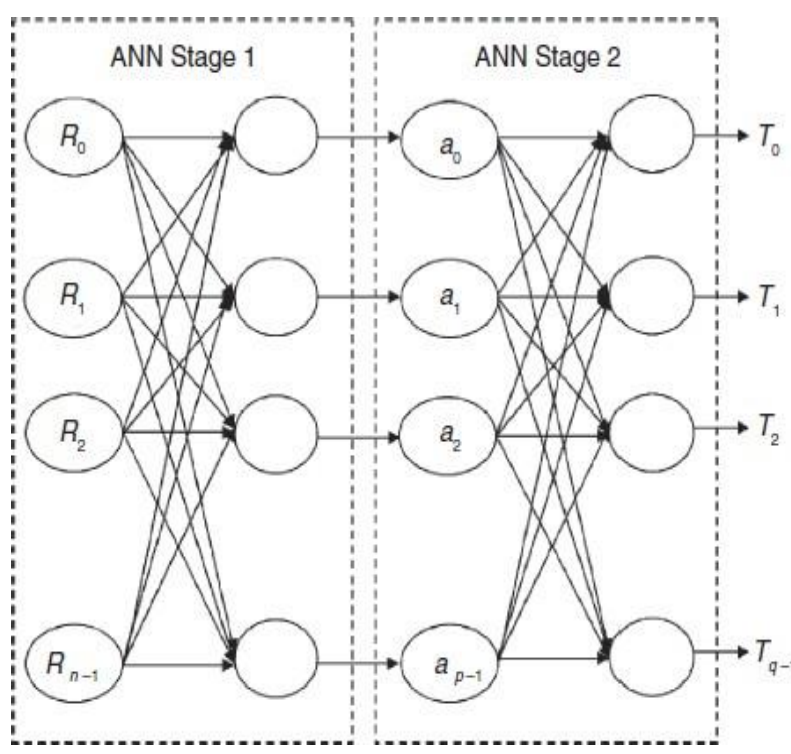

Fig 4: Two stage artificial neural network

\section{RESULTS}

To verify the feasibility, Physionet database [13] was used. Results of LMS algorithm and neural network based adaptive filter are shown in Fig.6.and Fig.7 respectively. The three different waveforms occurred from bottom to top are Fetal ECG, thoracic signal and abdominal signal.

Fig.8 illustrates the MSE for both the schemes which prove the proficiency of neural network based adaptive filter over LMS algorithm

The SNR graph of both the schemes is shown in Fig.9. While SNR increases, the signal strength also increases. Neural network based adaptive filter has better performance than LMS algorithm to filter out noise. To sum up, these results prove that neural network enhances performance over LMS algorithm 

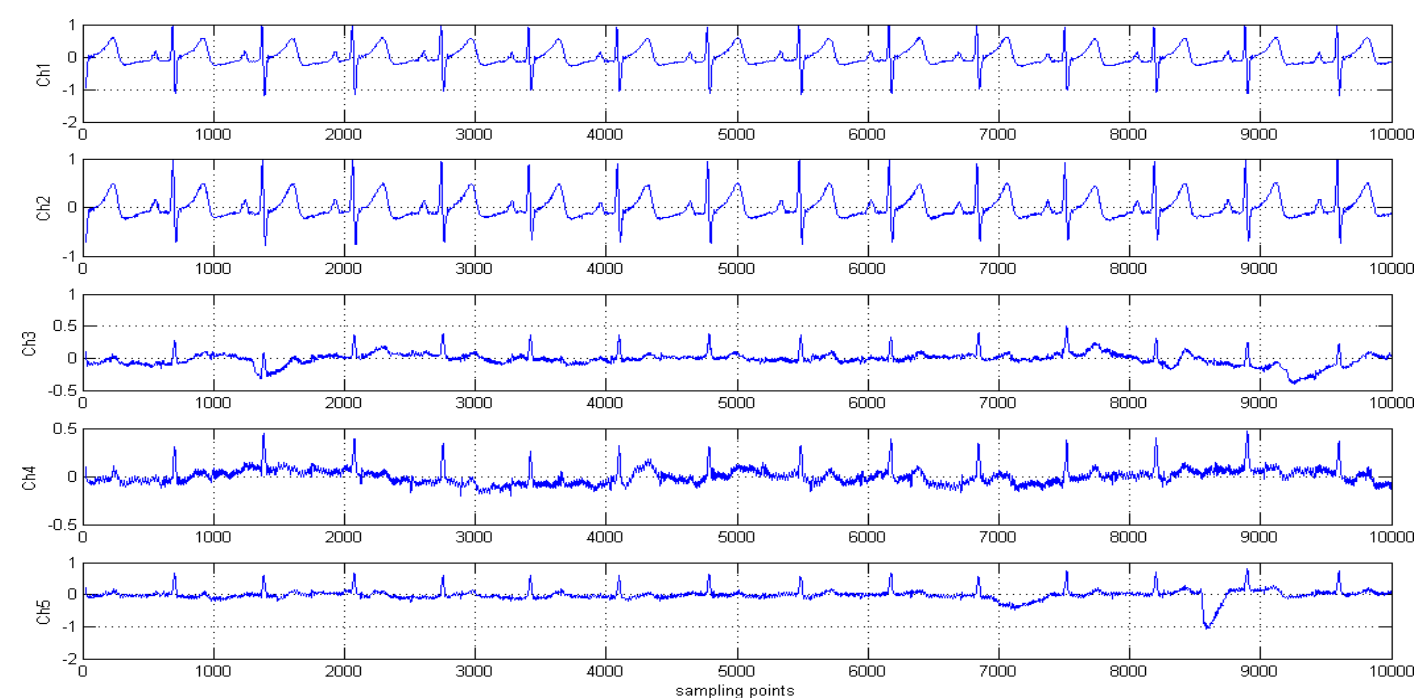

Fig 5: From a pregnant woman, five channels of ECG signals were composed. Where from Channels (chi) 1-2 are thoracic signals while Channels 3-5 are abdominal signals

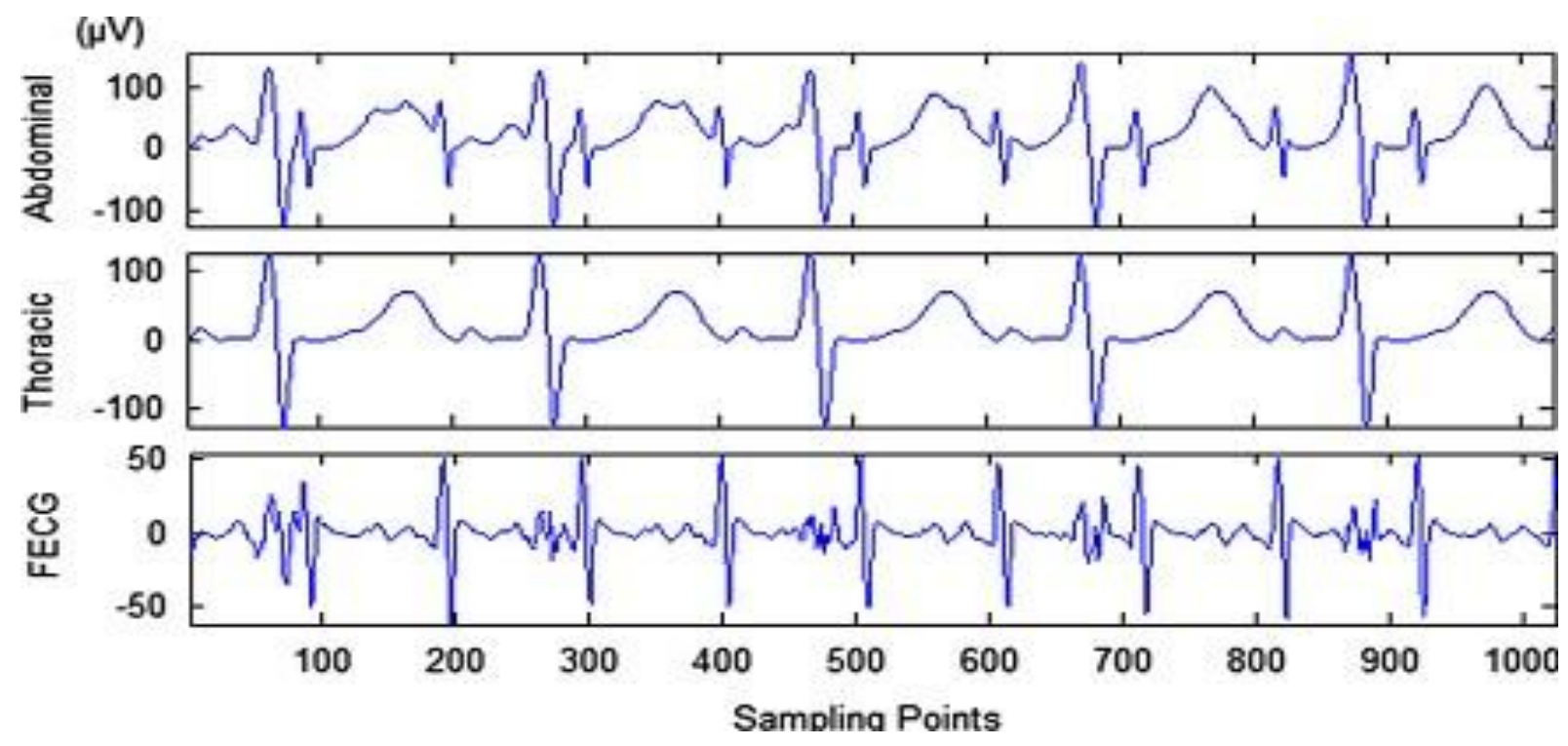

Fig 6: FECG extraction using LMS algorithm 

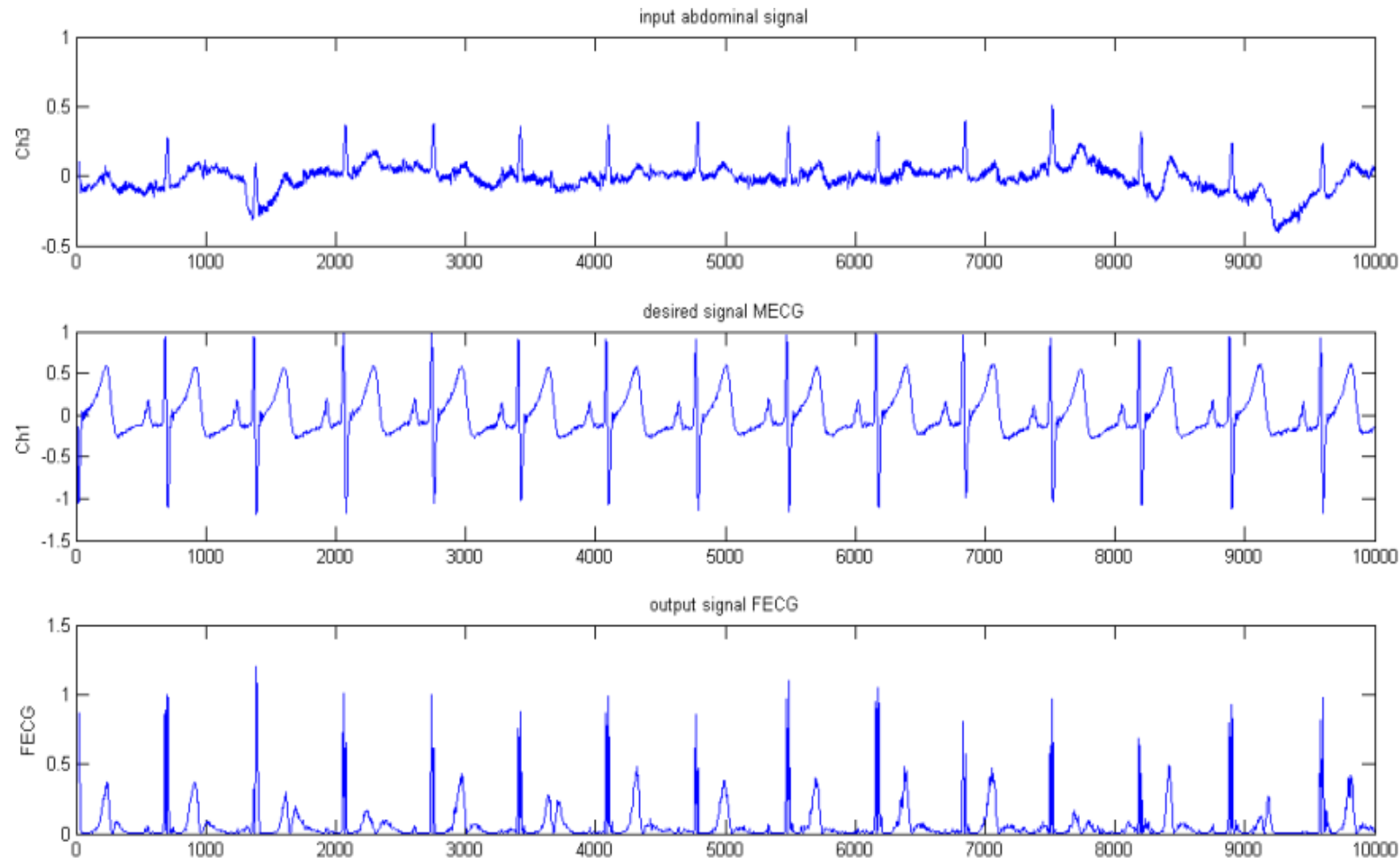

Fig 7: FECG extraction using neural network configuration

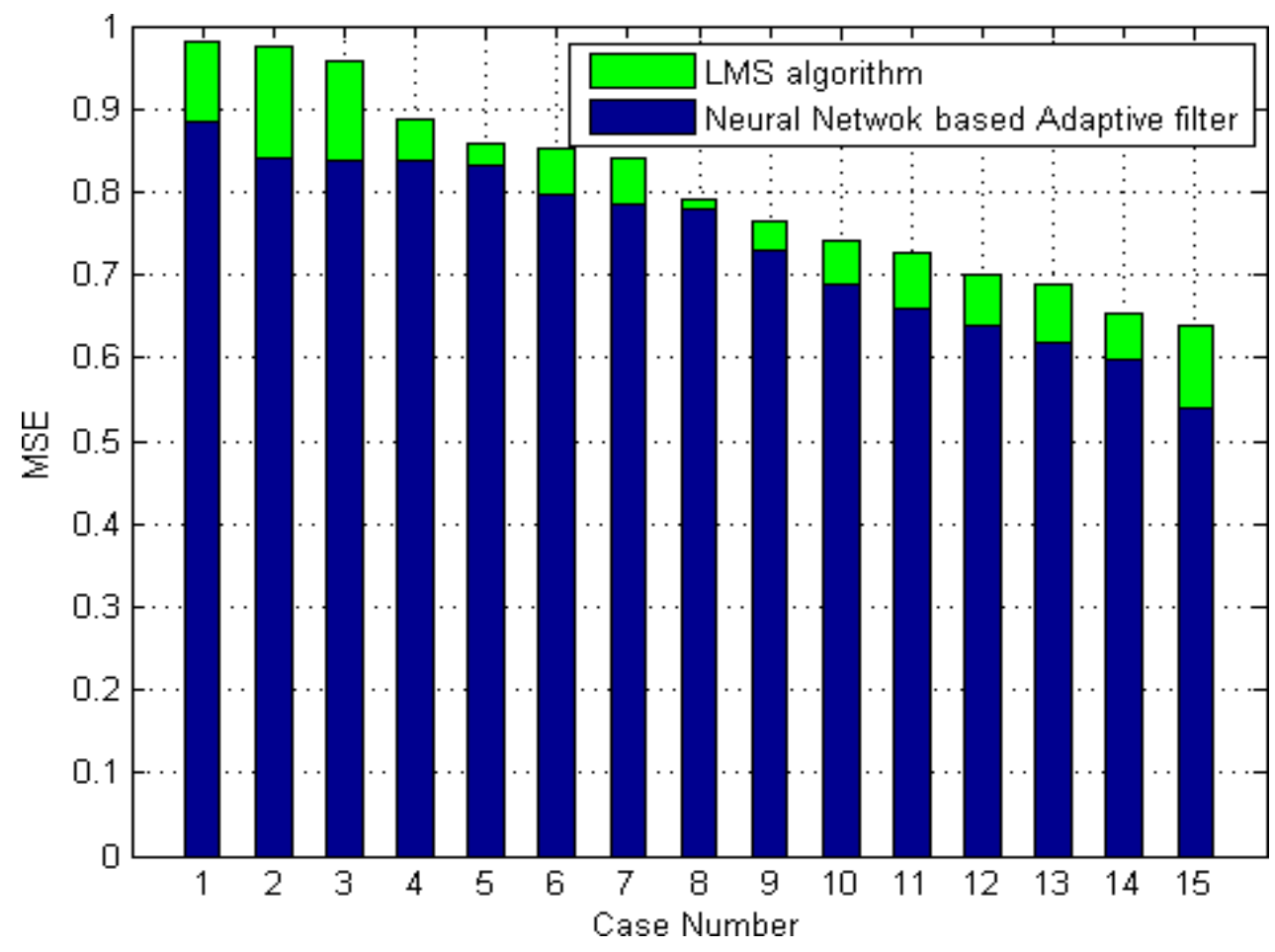

Fig 8: Comparison of MSE of LMS algorithm and neural network based Adaptive filter 


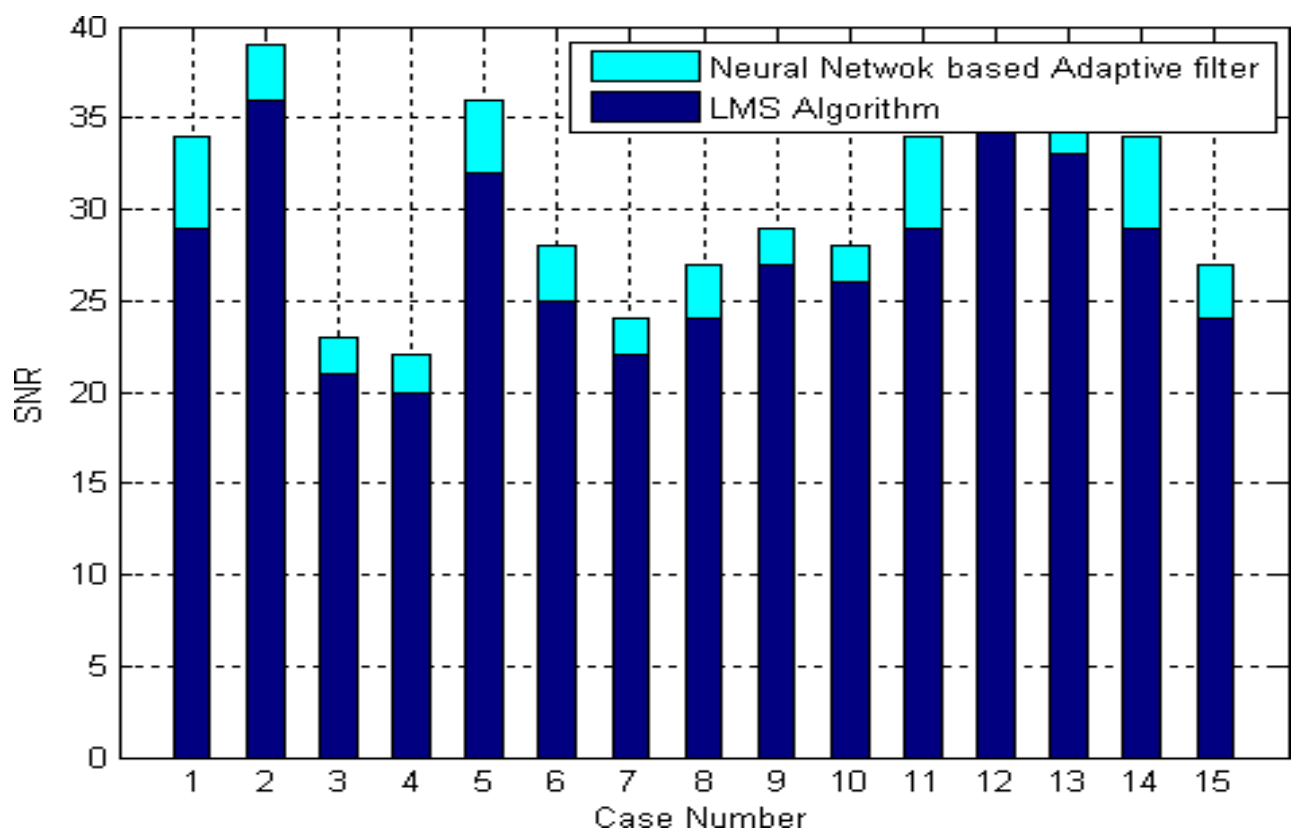

Fig 9: Comparison of SNR of LMS algorithm and neural network based Adaptive filter

\section{CONCLUSION}

The withdrawal of FECG is crucial from medical point of view to get tried and true data on fetal status. The extraction of clean FECG without interference is a daunting task. Neural network based adaptive filter is a versatile powerful technique for FECG extraction. Its performance has been proved quantitatively by examining SNR and MSE by using clinical data

\section{REFERENCES}

[1] D.J. Jagannath and A. Immanuel Selvakumar, "Superior foetal electrocardiogram signal elicitation using a novel artificial intelligent Bayesian methodology," Article Of Elsevier- Applied Soft Computing (In Press) Author, F.: Article title. Journal 2(5), 99- 110 (2016).

[2] M. Akay and E. Mulder, "Examining fetal heart-rate variability using matching pursuits", IEEE Engineering in Medicine and Biology, vol. 15 (5), pp. 64-67, 1996.

[3] L.D. Lathauwer, B.D. Moor, J. Vandewalle, Fetal electrocardiogram extraction by blind source subspace separation, IEEE Trans. Biomed. Eng. 47 (2000)567572.

[4] J. Szalai, F.E. Mozes, Determining fetal heart rate using independent component analysis, IEEE Intell. Comput. Commun. Process. . (ICCP) (2014) 11-16.

[5] A. Karimi Rahmati, S.K. Setarehdan, B.N. Araabi, A PCA/ICA based fetal ECG extraction from mother abdominal recordings by means of a novel data-driven approach to fetal ECG quality assessment, J. Biomed. Phys. Eng. 7(1) (2017) 37-50.
[6] H. Zhang, Z. Shi,C. Guo, E. Feng, Semi blind source extraction algorithm for fetal electrocardiogram based on generalized autocorrelations and reference signals, J.Comp. Appl. Math. 223(2009)409-420.

[7] B. Widrow, J.R. Glover, J.M. McCool, et al., Adaptive noise cancelling: principles and applications, Proc. IEEE 63 (12) (1975) 1692-1716.

[8] Wei Zheng, Xiaolong Li, Xueyun Wei, H. Hongxing, Foetal ECG Extraction by support vector regression, Electron. Lett. 52 (7) (2016) 506-507.

[9] P.P. Kanjilal, S. Palit, G. Saha, Fetal EXG extraction from single channel maternal ECG using singular value decomposition, IEEE Trans. Biomed. Engg.44 (1997)5159 .

[10] R. Almeida, H. Goncalves, J. Bernardes, A.P. Rocha, Fetal QRS detection and heart rate estimation: a waveletbased approach, Physiol. Meas. 35 (8) (2014)1723-1735.

[11] Mohammad Niknazar, Bertrand Rivet, Christian Jutten, Fetal ECG extraction by extended state kalman filtering based on single-Channel recordings, IEEE Trans. Biomed. Eng. 60 (5) (2013) 1345-1352.

[12] A. K. Barros and N. Ohnishi, "Fetal heart rate variability extraction by frequency tracking", in Proc. of the 3rd Intl. Conf. Independent Component Analysis and Source Separation, San Diego, CA, USA, 2001.

[13] http://www.physionet.org/pn3/nifecgdb. 